\title{
Impact of Propagation Environments on Emergency Message Dissemination in VANETs
}

\author{
Chakkaphong Suthaputchakun, \\ Department of Computer Engineering, Bangkok University \\ Khlong Luang, Pathum Thani, 12120, Thailand \\ E-mail: chakkaphong.s@bu.ac.th
}

\author{
Zhili Sun, and Mehrdad Dianati \\ Department of Electronic Engineering, University of Surrey \\ Guildford, Surrey, GU2 7XH, UK \\ E-mail: \{z.sun, and m.dianati\}@surrey.ac.uk
}

\begin{abstract}
In this paper, we investigate the impacts of different radio propagation environments on the performance of emergency message dissemination Vehicular Ad hoc Networks (VANETs). We compared the performances of the benchmark existing broadcast protocols for Emergency Message Dissemination in VANETs. We consider three different propagation models, namely, Log-Normal Shadowing, LongleyRice, and Nakagami to model six different simulation scenarios of both highway and urban areas. The objective is to provide a qualitative assessment of the protocols applicability in different vehicular scenarios. It is demonstrated that Trinary Partition Black-Burst based Broadcast Protocol (3P3B) reduces the communication delay, increases dissemination speed, increase reliability, and outperforms the well-known existing broadcast protocols for emergency message dissemination in VANETs in all propagation environments. The benchmark protocols achieve high performance in various vehicular scenarios both in highway and urban areas. However, there is still some reliability issue needed to be addressed by all existing protocols, such as communications in a very crowded city where the received communication signal is strongly distorted.
\end{abstract}

Keywords: Emergency Message, Multi-hop Broadcast Protocol, Priority, Trinary Partition, Mini-slot DIFS, VANETs, Propagation Model.

\section{INTRODUCTION}

Intelligent Transport Systems (ITS) applications aim to improve safety, efficiency, and comfort of land transport systems. In particular, researchers in both automotive industry and academic institutions [1-9] have been working to deploy emergency message (EM) dissemination applications over Vehicular Ad hoc Networks (VANETs).

Emergency message dissemination is a challenging problem in VANETs due to the lack of infrastructure support. A rapidly changing network topology due to high mobility of the vehicles also results in high rate of communication link failure. To this end, some recently proposed protocols aim to tackle the aforementioned challenges. To evaluate how effective such protocols perform when they are implemented in the real world, most of the existing works rely on the evaluation based on the simulation. However, the performance of the real implementation may be different from the results of simulations, as most of the existing simulators consider very basic and simple propagation models [2-7].

Propagations of electromagnetic waves is governed and determined by the features of the environment such as the obstacles between the transmitter and the receiver. The choice of radio propagation models has a strong impact on the performance of each individual communication protocol. The received radio signal is normally drawn by a distribution in all transmissions. Different vehicular propagation environments, as the result, give different successful communication rates due to their different distributions taken into account.

Several papers evaluated and compared performance of different existing protocols. However, the realistic propagation models were not appropriately taken into account by such papers [2-6]. These simple simulations may lead to impractical and unreliable performance evaluation. Therefore, the contributions of this paper are to evaluate and compare the performance of the existing benchmark broadcast protocols, investigate their applicability with realistic propagation models representing various vehicular environments such as highway and urban areas, and suggest possible solutions to improve and overcome the shortcoming of the existing protocols.

The rest of the paper is organized as follows. A brief description of three radio propagation models is given in Section II. Then, the detail of the existing benchmark broadcasting protocols for emergency message dissemination in VANETs is provided in Section III. Section IV describes the simulation configurations as a methodology for performance evaluation and comparison. Finally, the impact of radio propagation models on the performances of broadcasting protocols in VANETs is investigated and concluded in Sections V and VI, respectively.

\section{RADIO PROPAGATION MODELS}

A more realistic radio wave propagation model is allowed based on different probabilistic models [8-9]. During each transmission, the received power is estimated from a distribution and makes more diversity of a distribution of successful communications. With a certain probability, two vehicles may not be able to communicate with each other even they are very close to each other. On the other hand, with another certain probability, other two vehicles which locate beyond communication ranges of each other may be capable of making a communication. These effects mainly depend on probabilistic models and their parameters.

Each propagation model implements a set of mathematical models to provide an increasing communication precision. 
Radio wave propagation models consist of three modules namely path loss, shadowing (slow fading), and fast fading [14]. Path loss represents the loss of power when the radio signal propagates in a free space. Shadowing represents the signal propagation path where there are some fixed obstacles. Finally, fast fading aims to characterize effects of multiple propagation distances, fast movements of communication units, and signal reflectors.

In this paper, three propagation models (Log-Normal Shadowing, Longley-Rice, and Nakagami) have been studied and compared. These three models are mainly implemented as propagation models in vehicular ad hoc network research both in highway and urban scenarios [8-9].

\section{A. Log-Normal Shadowing}

The Log-Normal Shadowing model is the multiplicative model. The model assumes that there are several multiplicative random factors attenuating the received radio signal. Therefore, the Log-Normal Shadowing model distributes the reception power in the logarithmic domain using a normal distribution with variance $\sigma$.

$$
P_{r}\left(d ; \sigma^{2}\right) \sim L N\left(P_{r_{\mathrm{det}}}(d), \sigma^{2}\right)
$$

where $P_{\text {rate }}$ is a deterministic model which can be used from either Eq. (2) as Free Space Model or Eq. (3) as Two-Ray Ground Model.

$$
\begin{aligned}
& P_{r_{\mathrm{det}}}=\frac{P_{t} G_{t} G_{r} \lambda^{2}}{(4 \pi)^{2} d^{\alpha} L} \\
& P_{r_{\mathrm{det}}}=\frac{P_{t} G_{t} G_{r} h_{t}^{2} h_{r}^{2}}{d^{4} L}
\end{aligned}
$$

where $P_{t}$ is the transmitted power, $G_{t}$ and $G_{r}$ are the gains of the transmitter and receiver antenna, $\lambda$ is the wavelength, $\alpha$ is the path loss exponent, $L$ is the system loss, and $h_{t}$ and $h_{r}$ are the heights of the transmit and receive antennas, respectively. Therefore, the received power can be determined as in Eq. (4).

$$
P_{r}=P_{r_{\mathrm{det}}}\left(d_{o}\right) \times 10^{P L(d)}
$$

The received power is given as a multiplication between the deterministic received power and a Power Loss $(P L)$ scale factor in $\mathrm{dB}$ which can be determined using Eq. (5).

$$
P L(d)=-10 \alpha \log 10\left(\frac{d}{d_{o}}\right)+X_{\sigma}
$$

TABLE I

DEFAUlt PARAMETER VALUES DIFFERENT VeHICULAR ENVIRONMENTS

\begin{tabular}{lll}
\hline \hline Scenarios & Propagation Models & Parameter Values \\
\hline \hline Highway1 & Log-Normal Shadowing & $\alpha=1.9$ and $\sigma=4$ \\
Highway2 & Longley-Rice & $\alpha=1.9$ and $k=5$ \\
Highway3 & Longley-Rice & $\alpha=1.9$ and $k=11$ \\
\hline Urban1 & Log-Normal Shadowing & $\alpha=2.0$ and $\sigma=5$ \\
Urban2 & Nakagami & $\alpha=2.0$ and $m=1$ \\
Urban3 & Nakagami & $\alpha=2.0$ and $m=2.5$ \\
\hline
\end{tabular}

\section{B. Longley-Rice}

The Longley-Rice model or Rice model aims to capture rapid fluctuations of received radio signal because of multipath fading. The Rice model actually models the reception powers following the Rayleigh distribution which models the situation when there is no LOS, and there are only multipath components exist. Therefore, the amount of the received signal has a density of Rayleigh. However, the Rice model also takes into account the positive effects of a Line Of Sight (LOS) path with a certain scale factor $k$. Based on the real vehicle-to-vehicle measurement [10], the Rician $k$ factor in this paper is set to $8 \mathrm{~dB}$. The Rician reception power can be determined as in Eq. (6).

$$
P_{r_{\text {Rice }}}(d)=P_{r}(d) \times \Psi(d)
$$

where $P_{r}(d)$ can be referred to Eq. (4) with the value of $P L(d)$ expressed in Eq. (7).

$$
P L(d)=-\alpha \log 10\left(\frac{d}{d_{o}}\right)
$$

The Rician PDF with a normal distribution, $\Psi(d)$, is given in Eq. (8).

$$
\Psi(d)=c\left[N\left(\sqrt{P_{r}(d)}, 1\right)+\sqrt{2 k}\right]^{2}+N\left(\sqrt{P_{r}(d)}, 1\right)^{2}(8)
$$

where $c=\frac{1}{2(k+1)}$.

\section{Nakagami}

The Nakagami model encompasses several other distributions as particular cases. The model is highly generic and more realistic in which it allows similarly to the signals to be approximate. A gamma distribution is used to model the radio reception power as follows.

$$
P_{r}(d ; m) \sim \operatorname{Gammd}\left(m, \frac{P_{r_{\mathrm{det}}}(d)}{m}\right)
$$

where $m$ is fading effect intensity.

The Nakagami model is a good propagation model for VANETs in urban area, since it takes into account an influence of different kinds of building walls on the received signal.

By referring to several papers [8-10] dealing mainly with different propagation models for different closely realistic vehicular environments such as urban and highway, the value of the factor of each scenario in this paper is set to the default values shown in Table I.

\section{BROADCAST PROTOCOLS FOR EMERGENCY MESSAGE DISSEMINATION IN VANETS}

Broadcast protocol is a basic scheme to disseminate data from one sender to all receivers. The broadcast scheme seems 
to be one efficient solution for data dissemination in a high mobility network, which needs a fully distributed solution such as in VANETs. The vehicle does not need maintenance of routing tables, and information of each individual vehicle, such as position, speed, etc. However, the drawback of this scheme is high bandwidth usage, high data collision and errors, and low throughput. There are several researches and proposals of efficient broadcast protocols for emergency message dissemination in VANETs. This section gives a summary of such works.

\section{A. Urban Multi-hop Broadcast (UMB)}

UMB aims to provide collision avoidance, channel utilization, and broadcast communication reliability in VANETs [2]. Request to Broadcast/Clear to Broadcast (RTB/CTB) handshake scheme is implemented to avoid a hidden terminal problem and allows only one receiver during each hop of communications. Vehicles obey CSMA/CA to transmit a RTB packet. All vehicles which receive RTB packet in the dissemination direction start a black-burst transmission for a time period as a proportion to the distance. The black-burst is a channel jamming signal to represent the existing of the sender. Each vehicle begins to listen to the channel immediately after finishing the black-burst transmission. The vehicle will become a message forwarder if the channel is idle then it will send CTB back to the source while the others do nothing. The source vehicle will send a broadcast packet upon the CTB reception. Because the further vehicles normally transmit longer black-burst, UMB, as expected, experiences large communication latency.

\section{B. Smart Broadcast (SB)}

SB has a goal to maximize the message progress and minimize communication delay [3]. Network is partitioned into sectors. Each vehicle is capable of sensing its own position and calculating its sector. Contention mechanism is used to select a message forwarder. The source vehicle sends a RTB packet. Each vehicle calculates its sector upon a receipt of the RTB. The corresponding Contention Window $(\mathrm{CW})$ is also calculated according to the vehicle's sector. It is noted that vehicles of different sectors will have different and nonoverlapping values of $\mathrm{CW}$ sets. The set of $\mathrm{CW}$ values of outermost sector (furthest to the source vehicle) is the smallest and the set of $\mathrm{CW}$ values of innermost sector (closest to the source vehicle) is the biggest. Consequently, vehicles in the outermost sector will have higher chance to become a message forwarder. After channel contention, a vehicle will transmit CTB packet and will become a forwarder. The source vehicle will broadcast data frame. Only the forwarder will rebroadcast such packet to the next communication hop. Then, the process will repeat.

\section{Priority based Routing Protocol in VANETs (PRP)}

PRP is mainly designed based on IEEE 802.11e, which has aims to provide fully decentralized broadcast protocol, quality of service (QoS) mechanism for different message priorities, and maximum message dissemination distance per hop [4]. Similarly, PRP implements contention mechanism for a selection of the next-hop forwarder as implemented in SB. However, this contention mechanism is also applied for message prioritization. Therefore, a difference between SB and PRP is that PRP is able to provide differentiated services for different priorities of messages. For example, urgent messages are transmitted sooner than other lower priority messages. However, PRP also has the same drawback of large performance gap as in SB when a size of network becomes larger.

\section{Binary-Partition-Assisted Broadcast protocol (BPAB)}

$\mathrm{BPAB}$ [5] aims to reduce and makes communication delay as constant as possible regardless the size of network. BPAB achieves a good message progress speed by selecting the furthest forwarder. This protocol deploys a combination of a binary partitioning as well as a novel contention mechanism. The binary partitioning scheme constantly divides the communication area into multiple partitions. Only vehicles in the furthest partition contend with each other during the forwarding phase in this scheme. Thus, collision rate is reduced and the contention duration is stabilized. It is also shown that BPAB demonstrates a good performance in terms of the average dissemination speed compared to the other protocols, such as UMB and SB [5]. However, the further study may be needed to confirm that the binary partitioning is the optimal solution among any $n$-partitioning mechanisms.

\section{E. Trinary Partition Black-Burst based Broadcast protocol (3P3B)}

3P3B is a cross-layer protocol between MAC and network layers [6]. 3P3B aims to provide prioritization scheme for very urgent EMs as well as provide a constantly fast selection of efficient forwarders. In addition, it does not require beacons, and operates in a decentralized manner, which means that it does not require information exchange among the vehicles as well as support from Road Side Units (RSUs). 3P3B as a result can work on the fly. It uses Global Positioning System (GPS) to provide the vehicle with the information about their own positions and to assist with time synchronization among the vehicles. 3P3B consists of two major schemes; Mini-DIFS and Trinary Partitioning schemes.

The mini-DIFS is used as an emergency message prioritization scheme to give higher priority to very urgent EMs; i.e., very urgent EMs are broadcasted before low priority EMs. The trinary partitioning, which has been proof as the optimal partitioning among any other $n$-partitioning mechanisms, is implemented as a forwarding vehicle selection scheme. It aims to select the furthest vehicle as a forwarder to make a huge spatial message progress within a constant duration. It also considers vehicles travelling in both directions. In the paper [6], 3P3B has been proof to outperform the other existing broadcast protocols, including $\mathrm{BABP}, \mathrm{UMB}$ and $\mathrm{SB}$, for emergency message dissemination in VANETs.

Therefore, both 3P3B and BPAB are good candidates of the broadcast protocols in VANETs and have been chosen for 
performance evaluations and comparisons in different radio wave propagation models in this paper.

\section{Simulation CONFIGURATIONS}

In this section, we conduct performance evaluations based on simulations using OMNeT++, which is one of the wellknown and strong simulators for communication research and provides diverse configurations to support diffetent types of wired and wireless communication networks. Therefore, it also supports serveral sotiphicated radio wave propoagation models in wireless communications including Log-Normal Shadowing, Longley-Rice, and Nakagami models. In addition, to ensure the correctness of such models, the models in OMNeT++ have been cross validation checked against the other well-accepted simulators, such as NS-2 [13].

The simulation configuration is illustrated in Figure 1. The simulation area is set to a straight $40 \mathrm{~km}$-long highway, where the vehicles are randomly placed in the simulated highway. All vehicles move with random average speed varied from 60 to $100 \mathrm{~km} / \mathrm{hr}$. The number of vehicles need to transmit emergency messages simultaneously is 3 vehicles of which the emergency message frequency is varied from 0.1-0.3 Hz. There are 10 anchor vehicles placed at every $2 \mathrm{~km}$ following the transmitters. Therefore, the anchor vehicles cover the distance up to $20 \mathrm{~km}$ from the transmitters. It is note that all anchor vehicles only act as sink nodes to measure performance of the benchmark broadcast protocols at different distances from the transmitters. They do not participate in forwarding any emergency messages at all. To keep the distance between each anchor vehicle and transmitters constant, all of them move with the same average speed of $80 \mathrm{~km} / \mathrm{hr}$.

In the simulations, the vehicle density is varied from 5 to 30 vehicles per $\mathrm{km}$. The maximum speed limit on the highway is assumed to be $100 \mathrm{~km} / \mathrm{hr}$. [12]. The paper compares performance of 3P3B against BPAB as both of them are good benchmark broadcast protocols in VANETs. To the best comparison, both protocols treats all transmitting EMs as AC_VO (Voice Access Category) which is the highest prioritized data packets according to IEEE802.11p standard [11] shown in Table II. The number of iterations, $N$, and contention window, $c w$, used in both binary and trinary partitioning schemes are set to $(4,5),(3,2)$ for $\mathrm{BPAB}$ and 3P3B, respectively, as they are optimal values presented in [6]. All other default parameter values used in the simulations are summarized in Table III.

\section{PERFORMANCE EVALUATION RESULTS AND ANALYSIS}

In this section, we present performance evaluation results in terms of packet delivery ratio, one-hop delay, and message dissemination speed of both benchmark protocols in different vehicular scenarios modeled by different radio propagation models.

\section{A. Packet Delivery Ratio}

The packet delivery ratio is the percentage between the total number of successfully received emergency messages and the total number of transmitted emergency messages at different dissemination distances from 2 to $20 \mathrm{~km}$. The result in term of the packet delivery ratios of both $3 \mathrm{P} 3 \mathrm{~B}$ and $\mathrm{BPAB}$ in different scenarios are shown in Fig. 2(a) and 2(b). In this simulation, the vehicle density is set to 20 vehicles per $\mathrm{km}$.

In the highway scenarios, the results are really close to each other regardless the different propagation models. When the emergency messages are disseminated further, the delivery ratios of both $3 \mathrm{P} 3 \mathrm{~B}$ and $\mathrm{BPAB}$ are decreased. The protocols attain roughly more than $95 \%$ in term of packet delivery ratio for the communication within the first $2 \mathrm{~km}$ in the highway scenarios. However, at the largest communication distance of $20 \mathrm{~km}$, both protocols can only achieve at least $65 \%$ in the worst case and achieve up to $80 \%$ in the best case. This is very acceptable according to the huge communication distance of $20 \mathrm{~km}$. By comparing the delivery ratio of both benchmark protocols, 3P3B outperforms $\mathrm{BPAB}$ in all cases regardless the dissemination distances and the propagation models.

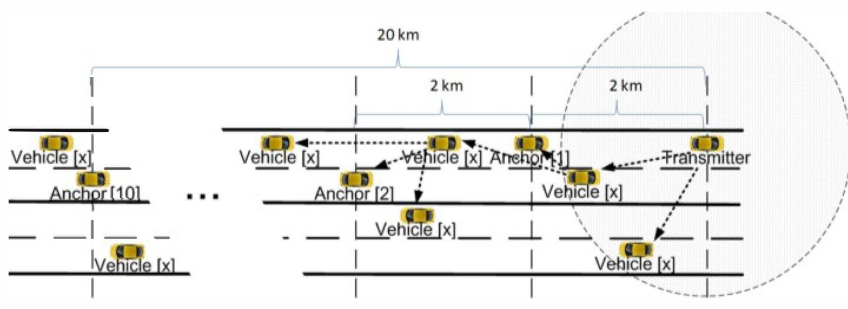

Fig. 1 Simulation scenario

TABLE II CONTENTIONPARAMETER VALUES OF IEEE802.11P STANDARD

\begin{tabular}{ccccc}
\hline \hline AC & CWmin & CWmax & AIFSN & TXOP \\
\hline AC_BK & aCWmin & aCWmax & 9 & 0 \\
AC_BE & aCWmin & aCWmax & 6 & 0 \\
AC_VI & $(a C W m i n+1) / 2-1$ & aCWmin & 3 & 0 \\
AC_VO & $(\mathbf{a C W m i n}+\mathbf{1}) / \mathbf{4 - 1}$ & $(\mathbf{a C W m i n}+\mathbf{1}) / \mathbf{2}-\mathbf{1}$ & $\mathbf{2}$ & $\mathbf{0}$ \\
\hline
\end{tabular}

TABLE III

Other Default Parameter Values of The Simulations

\begin{tabular}{ll}
\hline \hline Parameters & Default Values \\
\hline \hline Standards & IEEE 802.11p/ IEEE1609.4 \\
Communication Frequency & $5.9 \mathrm{GHz}$ \\
Evaluated Protocols & BPAB and 3P3B \\
Propagation Model & Log-Normal Shadowing, Longley- \\
& Rice, and Nakagami \\
Transmission Power $\left(P_{t}\right)$ & $20 \mathrm{~mW}$ \\
Sensitivity & $-94 \mathrm{dBm}$ \\
Thermal Noise & $-110 \mathrm{dBm}$ \\
Transmitter Antenna Gain $\left(G_{t}\right)$ & $0 \mathrm{~dB}$ \\
Receiver Antenna Gains $\left(G_{r}\right)$ & $0 \mathrm{~dB}$ \\
Bit Rate & $18 \mathrm{Mbps}$ \\
Emergency Message Packet Frequency & $0.1-0.3 \mathrm{~Hz}$ \\
Emergency Message Packet Size & $500 \mathrm{Bytes}$ \\
RTB Packet Size & $20 \mathrm{Bytes}$ \\
CTB Packet Size & $14 \mathrm{Bytes}$ \\
Slot Time & $13 \mu \mathrm{s}$ \\
DIFS & $58 \mu \mathrm{s}$ \\
SIFS & $32 \mu \mathrm{s}$ \\
Max Channel-Propagation Delay & $2 \mu \mathrm{s}$ \\
Transceiver's Switching Time & $1 \mu \mathrm{s}$ \\
Maximum Speed Limit on the Highway & $100 \mathrm{~km} / \mathrm{hr}$. \\
Number of Transmitters & 3 \\
Vehicle Density & $5-30 \mathrm{vehicle} / \mathrm{km}$ \\
$(N, c w)$ of BPAB & $(4,5)$ \\
$(N, c w)$ of 3P3B & $(3,2)$ \\
\hline
\end{tabular}




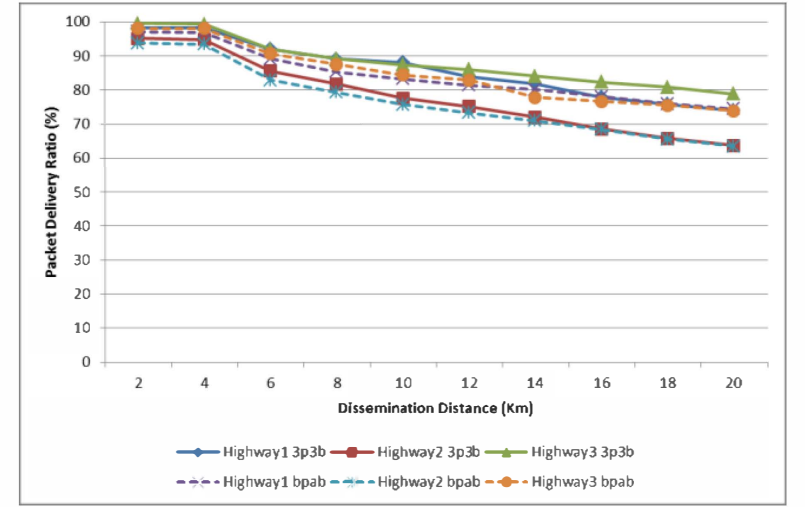

(a) Simulation results in terms of packet delivery ratio in highway scenarios

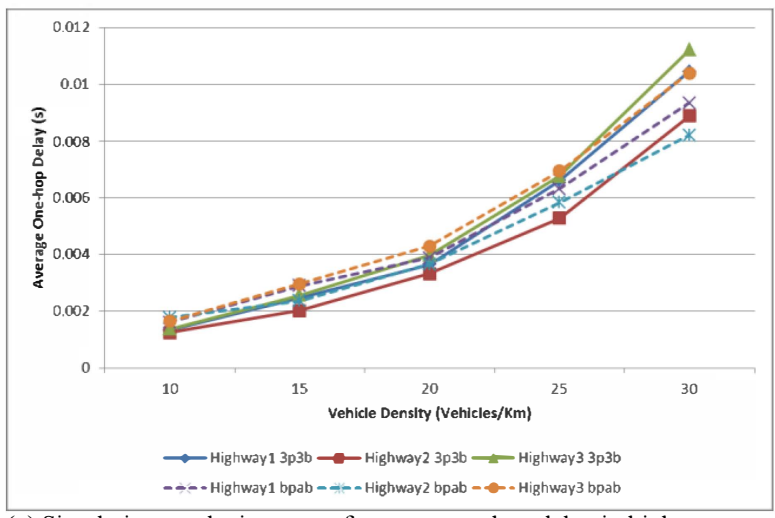

(c) Simulation results in terms of average one-hop delay in highway scenarios

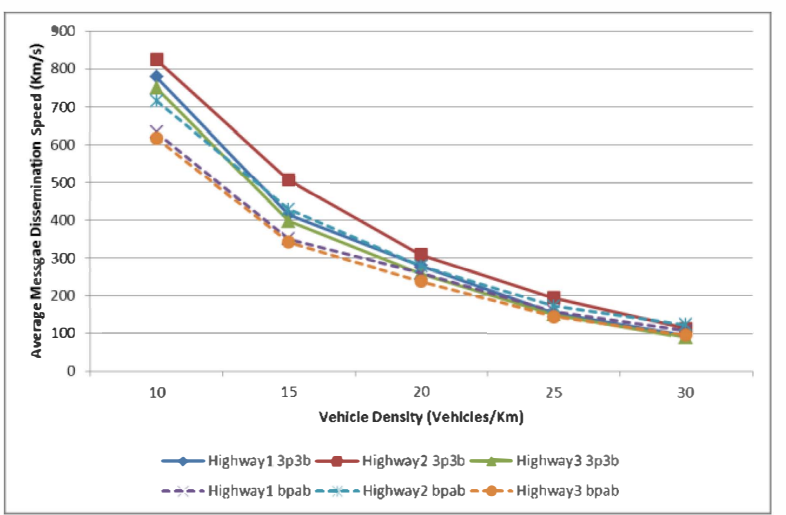

(e) Simulation results in terms of average dissemination speed in highway scenarios

Fig. 2 Simulation results in terms of packet delivery ratio, averagec

In the urban scenarios, the degradation in term of delivery ratio when the dissemination is larger becomes more significant compared to that in the highway scenarios. This is due to a too-strong influence of the building taken into account on the received signal modeled by Nakagami propagation model. Within the first $2 \mathrm{~km}$, both 3P3B and $\mathrm{BPAB}$ achieve nearly $90 \%$ in term of packet delivery ratio regardless the scenarios. However, the Urban3 scenario modeled with Nakagami presents the worst case scenario in urban with only $10 \%$ delivery rate at $20 \mathrm{~km}$ distance. This may be considered too-extreme scenario. By comparing to the other 2 scenarios (Urban1 and Urban2) the delivery ratios of

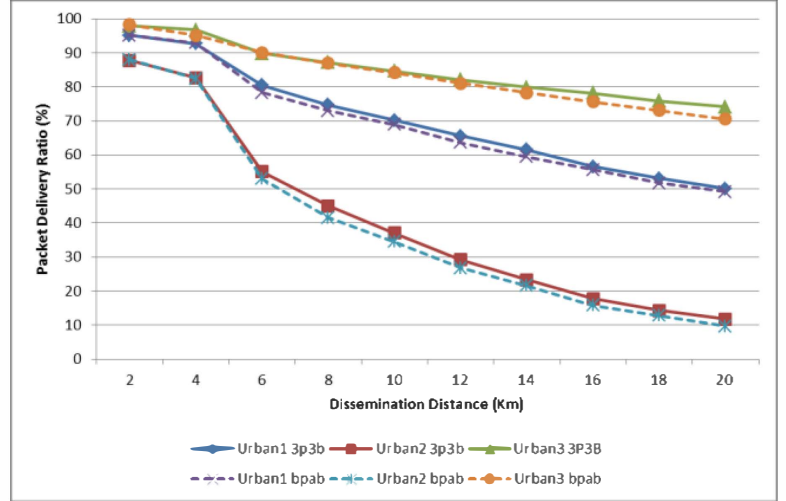

(b) Simulation results in terms of packet delivery ratio in urban scenarios

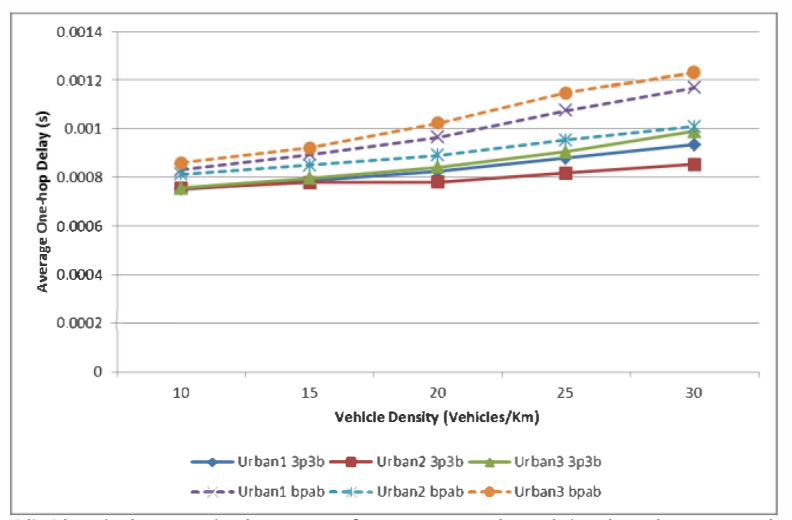

(d) Simulation results in terms of average one-hop delay in urban scenarios

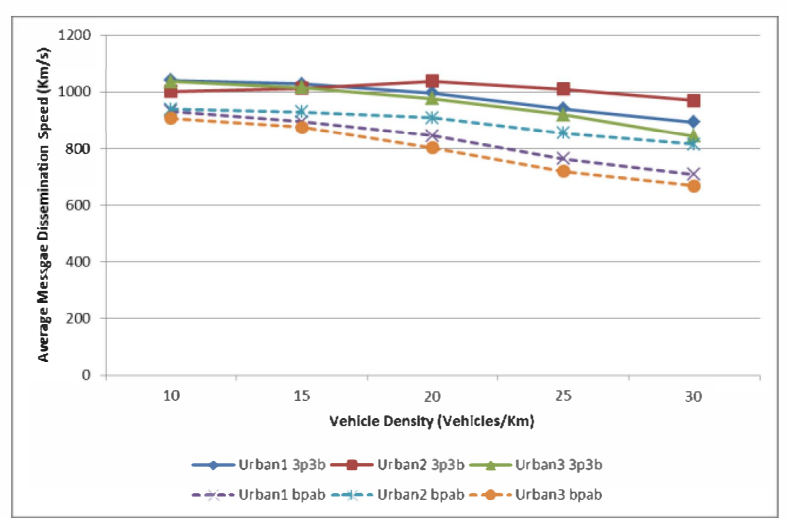

(f) Simulation results in terms of average dissemination speed in urban Scenarios 
delay in both highway and urban scenarios are shown in Fig. 2(c) and 2(d), respectively. Regardless the different scenarios and vehicle density, 3P3B outperforms BPAB by achieving lower one-hop delay in most cases. In addition, the achieved one-hop delay by both benchmark protocols is only in $m s$.

Surprisingly, the communication in urban experiences roughly 10 times lower in term of one-hope delay compared to the highway scenarios. As previously presented, there is higher rate of communication failure in the urban scenarios. Therefore, the higher number of messages have been discarded and dropped in the urban cases. Compared to the highway scenarios, both protocols try their best to delivery messages even with longer delay. Therefore, they achieve higher delivery ratio with the tradeoff of the larger communication delay as it can be observed in Fig. 2(a)-(d).

\section{Average Message Dissemination Speed}

The average message dissemination speed is a distance covered by an emergency message in one second. Fig. 2(e) and 2(f) illustrate performance comparison in term of the average message dissemination speed between 3P3B and $\mathrm{BPAB}$ in both highway and urban scenarios modeled by different propagation models. In most cases regardless the propagation models, 3P3B outperforms $\mathrm{BPAB}$ by giving the faster dissemination speed.

In addition, the dissemination speed achieved in the urban scenarios is higher than that in the highway, especially at the high vehicle density. Because a lot of messages have been retransmitted several times to reach all receivers, the dissemination speed is compromised in the highway scenarios. In contrast, in the urban scenarios, most of messages are dropped after several unsuccessful retransmissions and these dropped messages are excluded from the statistic collection in term of the dissemination speed. Therefore, the dissemination speed of the message in the urban scenarios seems to be better than the highway scenarios. However, if all dropped messages are included in the statistic, the performance of the urban scenarios would be far worse than the highway scenario.

\section{CONCLUSION}

This paper evaluates and compares the performance of both benchmark broadcasting protocols; i.e. 3P3B and BPAB, for emergency message dissemination in VANETs using three different and well-accepted radio propagation models such as Log-Normal Shadowing, Longley-Rice, and Nakagami models. These models are configured with different parameter values to reflect the different vehicular communication environments, such as highway and urban scenarios. Both $3 \mathrm{P} 3 \mathrm{~B}$ and $\mathrm{BPAB}$ perform really well by achieving roughly $95 \%$ and nearly $90 \%$ in term of packet delivery ratio in the highway and urban scenarios, respectively. At the largest distance of $20 \mathrm{~km}$, the protocols achieve $65 \%$ - $80 \%$ delivery ratios in the highway, and $60 \%-70 \%$ in the urban. Additionally, 3P3B in all cases outperforms BPAB in terms of dissemination speed, communication delay, and successful delivery ratio. Therefore, 3P3B becomes a promising candidate broadcast communication in VANETs.
However, in a very crowded urban area where there are a lot of huge buildings surrounding the communication, which can be modeled by the Nakagami as Urban3, the performance evaluation shows the possible shortcoming of all benchmark protocols in term of packet delivery ratio. Since there is a strong influence of dense buildings on received signal, such as signal scattering, reflection, and diffraction, some additional mechanisms such as store-carry-forward may be required by both protocols, to improve the reliability of the communication, particularly in the city center before their real implementations in the future.

\section{ACKNOWLEDGMENT}

This work is supported by Bangkok University, FP7 Mission, ESA SatNex III, and CATT No. 2007 DQ305156.

\section{REFERENCES}

[1] "DRIVE C2X", [Online]. Available: http://www.drive-c2x.eu.

[2] G. Korkmaz, E. Ekici, F. Ozguner, and U. Ozguner, "Urban multi-hop broadcast protocol for inter-vehicle communication systems", in Proc the 1st ACM international workshop on Vehicular ad hoc networks Philadelphia, 2004, pp. $76-85$

[3] E. Fasolo, A. Zanella, and M. Zorzi, "An Effective Broadcast Scheme for Alert Message Propagation in Vehicular Ad hoc Networks", in Proc. IEEE International Conference on Communications, Istanbul, 2006 pp. $3960-3965$

[4] C. Suthaputchakun, and Z. Sun, "Priority based Routing Protocol in Vehicular Ad hoc Network", 2011 IEEE Symposium on Computers and Communications, Kerkyra, 2011, pp.723-728.

[5] J. Sahoo, E.H.-K. Wu, P.K. Sahu, M. Gerla, "Binary-Partition-Assisted MAC-Layer Broadcast for Emergency Message Dissemination in VANETs", IEEE Transactions on Intelligent Transportation Systems, 2011, vol.12, no.3, pp.757-770.

[6] C. Suthaputchakun, M. Dianati, and Z. Sun, "Trinary Partition BlackBurst based Broadcast Protocol for Time-Critical Emergency Message Dissemination in VANETs," IEEE Transactions on Vehicular Technology, 2014, vol.63, no.6, pp.2926-2940.

[7] K. Ramachandran, M. Gruteser, R. Onishi, and T. Hikita, "Experimental analysis of broadcast reliability in dense vehicular networks," IEEE Vehicular Technology Magazine, 2007, vol. 2, no. 4, pp.26-32.

[8] U. Luis, T. Carolina, M. Isabel, and A. Monica, "Propagation and Packet Error models in VANET simulations", IEEE Latin America Transactions, 2014, vol. 12, no. 3, pp. 499-507.

[9] Rhattoy, A., and A. Zatni, "Impact of Mobility and Maps Size on the Performances of VANETS in Urban Area", International Journal of Computer Engineering \& Technology, 2013, vol. 4, no. 2, pp. 556-568

[10] J.S. Davis, and J.P.M.G. Linnartz, "Vehicle to vehicle RF propagation measurements", in Proc. 1994 Conf erence Record of the Twenty-Eighth Asilomar Conference on Signals, Systems and Computers, CA, 1994, vol. 1 , pp. $470-474$

[11] "802.11 p-2010 - IEEE Standard for Information technology-- Local and metropolitan area networks-- Specific requirements-- Part 11: Wireless LAN Medium Access Control (MAC) and Physical Layer (PHY) Specifications Amendment 6: Wireless Access in Vehicular Environments", IEEE Std 802.11p-2010, 2010, pp.1-51.

[12] The Highway Code. [Online]. Available https://www.gov.uk/browse/driving/highway-code.

[13] A. Kuntz, F. Schmidt-Eisenlohr, O. Graute, H. Hartenstein, and M Zitterbart, "Introducing probabilistic radio propagation models in omnet++ mobility framework and cross validation check with ns-2", in Proc. the 1st international conference on Simulation tools and techniques for communications, networks and systems \& workshops, Belgium, 2008, pp. 1-7.

[14] A. Schmitz, and M. Wenig, "The effect of the radio wave propagation model in mobile ad hoc networks", in Proc. the 9th ACM international symposium on Modeling analysis and simulation of wireless and mobile systems, New York, 2006, pp. 61-67 